\title{
Bilateral acute angle-closure glaucoma following tramadol subcutaneous administration
}

\author{
Anis Mahmoud ${ }^{1 *}$, Fatma Abid ${ }^{1}$, Imen Ksiaa², Sourour Zina ${ }^{2}$, Riadh Messaoud ${ }^{1}$ and Moncef Khairallah ${ }^{2}$
}

\begin{abstract}
Background: To report a case of bilateral acute angle closure-glaucoma following the use of subcutaneous Tramadol.

Case presentation: A 42-year-old healthy man with unremarkable past medical and ocular history, was admitted to the Orthopedic Department for surgical treatment of a bilateral open fracture of the femur following a road accident. Three hoursafterTramadolsubcutaneous injection, the patient complained of a bilateral acute painful visual loss with persistent vomiting. An ocular examination showed bilateral acute angle-closure-glaucoma. The patient was treated with topical anti-glaucoma therapy and intravenous Mannitol 20\%.After resolution of ocular hypertension attack, NdYag laser peripheral iridotomy was performed on both eyes. After a follow-up period of 7 days visual acuity improved to 20/20 in both eyes and intraocular pressure returned to normal levels.

Conclusions: This case highlights the risk of developing bilateral acute angle-closure glaucoma after Tramadol administration.
\end{abstract}

Keywords: Angle closure glaucomaMydriasisTramadolAnterior segment OCT

\section{Background}

Acute angle-closure glaucoma (AACG) is one of the most vision-threatening conditions being the leading cause of bilateral blindness in the Asian population [1].

Several types of drugs may precipitate bilateral AACG such as adrenergic, anticholinergic [2] and sulfonamidederived medications (diuretic, antibiotics, antiepileptic and antidepressant) $[3,4]$.

Herein, we report a case of bilateral AACG following Tramadol subcutaneous administration.

\section{Methods}

It was a single case report. Written informed consent was obtained from the patient before publishing.

\section{Case presentation}

A 42-year-old healthy male was admitted to the Emergency Department after a road accident. He was referred

\footnotetext{
* Correspondence: mahmoudanisss@hotmail.fr

${ }^{1}$ Department of Ophthalmology, Tahar Sfar University Hospital, Mahdia;

Faculty of Medicine, University of Monastir, Monastir, Tunisia

Full list of author information is available at the end of the article
}

to the Orthopedic Department for management of a bilateral open fracture of the femur.

To deal with acute pain, the patient received a subcutaneous injection of $100 \mathrm{mg}$ of Tramadol after which he experienced a transient bilateral blurred vision that resolved spontaneously. Eight hours later, a second subcutaneous injection of $100 \mathrm{mg}$ of Tramadol was administered and $3 \mathrm{~h}$ later, the patient started complaining of severe blurred vision with headache and vomiting.

On examination,the visual acuity was $20 / 400$ in the right eye (RE) andwas limited to light perception in the left eye (LE). A slit lamp examination showed bilateral conjunctival injection associated with corneal edema. The anterior chambers were narrow and the pupils were mid-dilated with a sluggish light reaction in both eyes (Fig. 1). The intraocular pressure (IOP) with applanation tonometry was $46 \mathrm{mmHg}$ in the RE and $55 \mathrm{mmHg}$ in the LE. A gonioscopy showed a narrow angle with no visible structures. The fundus details were hidden by the corneal edema. An anterior segment optical coherence 

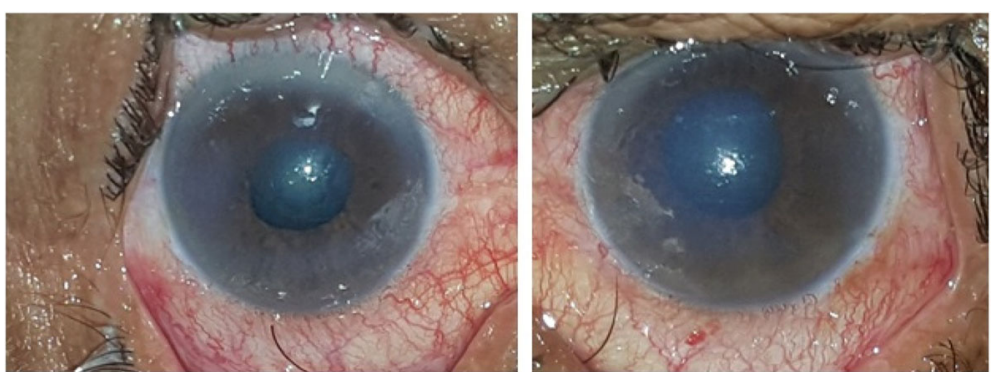

Fig. 1 Slit-lamp photograph showing conjunctival injection associated with corneal edema and mid-dilated pupil in both eyes

tomography (AS-OCT) performed during the acute phase demonstrated a closed iridocorneal angle with a markedly reduced anterior chamber depth of $1.9 \mathrm{~mm}$ (Fig. 2).

The diagnosis of bilateral drug-induced AACG in a predisposed patient was therefore made. The suspected culprit drug, Tramadol, was discontinued and intravenous mannitol 20\%, topical anti-glaucoma drops and pilocarpin eye drops were administered.

Twenty-four hours later, the pain resolved and IOP dropped to $16 \mathrm{mmHg}$ in both eyes. The pupilsreturned to normal size and were fully reactive. A second gonioscopy showed angles open to scleral spur without peripheral anterior synechiae.

On day three of follow-up, the corneas became clearer, IOP remained well-controlled and the fundus examination showed a hyperhemic optic disc with normal C/D ratio. Subsequently NdYag laser iridotomy was successfully performed on both eyes (Fig. 3). The final visual acuity was $20 / 20$ in both eyes and the intraocular pressure returned to normal levels.

\section{Discussion}

Acute angle closure-glaucoma (AACG) is a painful, potentially blinding disease. Many systemic drugs may precipitate bilateral acute angle closure attacks. The most common of these drugs are the -adrenergic (Adrenaline, Ephedrine) and anticholinergic drugs (Atropine) through pupillary block mechanism following mydriasis in predisposed individuals with narrow iridocorneal angles [5]. Ocular conditions that predispose to angle closure are high hyperopia, shallow anterior chamber, and thick lens [6].

Other drugs may produce bilateral AACG secondary to ciliochoroidal effusion with anterior rotation of the ciliary body and forward displacement of the lens-iris diaphragm [7, 8]. They include mainly sulfonamide derivatives and Topiramate $[2,7]$.

To the best of our knowledge, the association of Tramadol administration with bilateral acute angle closure has not been previously reported. Tramadol is a commonly prescribed opioid used in severe pain. Tramadol can cause miosis through stimulation of
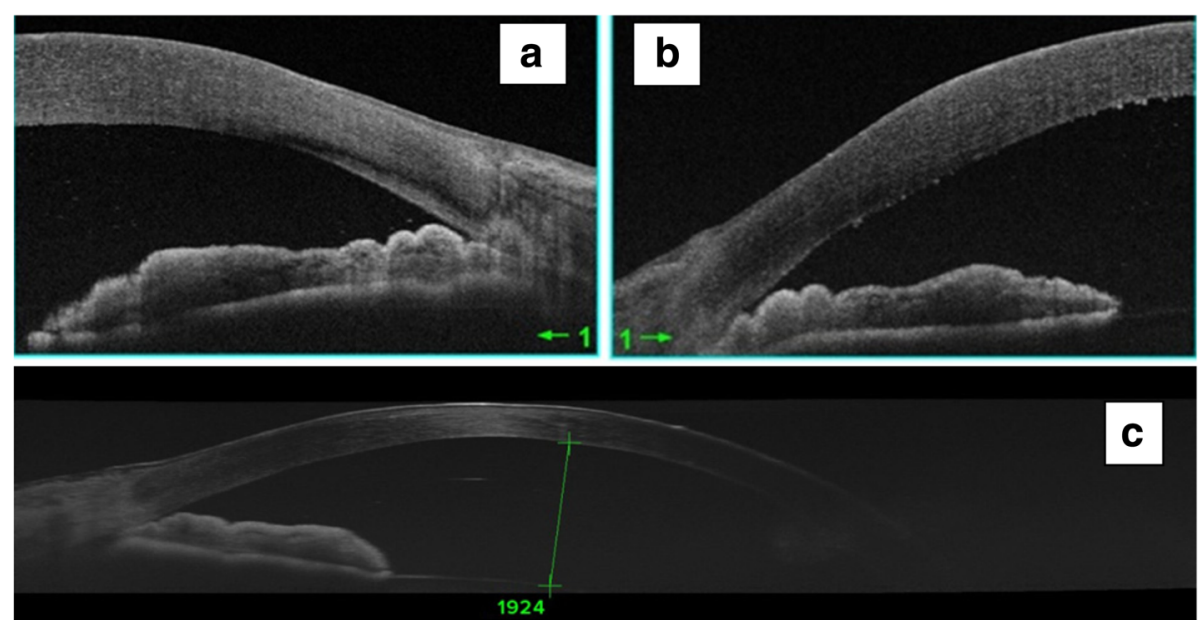

Fig. 2 AS-OCT performed at the acute phaseconfirms the angle closure in both eyes (a and $\mathbf{b}$ ). Note the presence of shallow anterior chamber (c) 


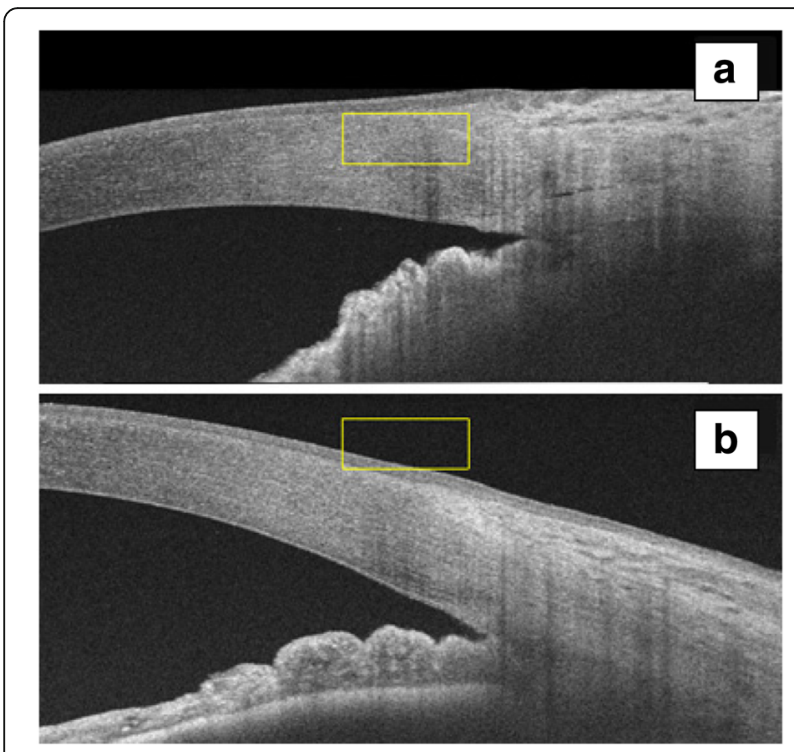

Fig. 3 AS-OCT performed $72 \mathrm{~h}$ after Nd-YAG laser iridotomy shows angle opening in both right (a) and left (b) eyes

opioid receptors [9] or mydriasis through stimulation of the adrenergic receptors [10]. In fact, it inhibits serotonin and noradrenaline reuptake from the synaptic cleft, and stimulates pre-synaptic release of serotonin [11]. Thus, the concentration of these two substances increases and so do their effects. The ubiquitous pupillary response to Tramadol may dependon individual metabolic abilities. In fact, miosis is more likely to occur in extensive Tramadol metabolisers while in intermediate and poor metabolisers, mydriasis might develop because of a delay in conversion of Tramadol to its active metabolite [12].

\section{Conclusion}

Tramadol administration may cause mydriasis which can precipitate an attack of acute AACG in predisposed individuals with shallow anterior chambers. All clinicians should be aware of the potential risk of AACG in patients treated with Tramadol.

\section{Abbreviations}

AS-OCT: Anterior segment optical coherence tomography; AACG: Acute angle-closure glaucoma; IOP: Intraocular pressure; LE: Left eye; RE: Right eye

\section{Acknowledgments}

Not applicable.

Funding

None.

\section{Availability of data and materials}

Data for this case report were collected by chart review of the patient's electronic medical record, which is not publicly available due to privacy considerations.

\section{Authors' contributions}

AM: acquisitionof data and drafting the manuscript. FA: analysis of dataand drafting the manuscript. IK: conception and revising the manuscript. SZ: acquisition of data and drafting the manuscript. RM: conception and revising the manuscript. MK: interpretation of data and revising the manuscript. All authors agreed to be accountable for all aspects of the work. All authors have read and approved the manuscript.

Ethics approval and consent to participate Not applicable.

\section{Consent for publication}

Informed written consent to publish data and images was obtained from the patient.

\section{Competing interests}

The authors declare no financial competing interests.

\section{Publisher's Note}

Springer Nature remains neutral with regard to jurisdictional claims in published maps and institutional affiliations.

\section{Author details}

'Department of Ophthalmology, Tahar Sfar University Hospital, Mahdia; Faculty of Medicine, University of Monastir, Monastir, Tunisia. ${ }^{2}$ Department of Ophthalmology, Fattouma Bourguiba University Hospital, Faculty of Medicine, University of Monastir, Monastir, Tunisia.

Received: 31 August 2017 Accepted: 12 February 2018

Published online: 17 February 2018

\section{References}

1. Foster PJ. The epidemiology of primary angle closure and associated glaucomatous optic neuropathy. SeminOphthalmol. 2002;17(2):50-8.

2. Senthil S, Garudadri C, Rao HBL, Maheshwari R. Bilateral simultaneous acute angle closure caused by sulphonamide derivatives: a case series. Indian J Ophthalmol. 2010;58:248-52.

3. Durai I, Mohan Dhavalikar M, Anand CP, Ganesh V, Krishnadas R. Bilateral, simultaneous, acute angle closure glaucoma in pseudophakia induced by chlorthalidone. Case Rep in Ophthalmol Med. 2016;3713818.

4. Chen SH, Karanjia R, Chevrier RL, Marshall DH. Bilateral acute angle closure glaucoma associated with hydrochlorothiazide-induced hyponatraemia. BMJ Case Rep. 2014:bcr2014206690.

5. Raj KM, Reddy PA, Kumar VC. Bilateral angle closure glaucoma following general anaesthesia. J PharmBioalliedSci. 2015;7(1):S70-1.

6. Lowe RF. Aetiology Of the anatomical basis for primary angleclosure glaucoma.Biometrical comparisons between normal eyes and eyes with primary angleclosure glaucoma. Br J Ophthalmol. 1970:54(3):161-9.

7. Fraunfelder FW, Fraunfelder FT, Keates EU. Topiramate-associated acute, bilateral, secondary angleclosure glaucoma.Ophthalmology 2004;111(1):109-111.

8. Medeiros FA, Zhang XY, Bernd AS, Weinreb RN. Angle-closure glaucoma associated with ciliary body detachment in patients using topiramate. Arch Ophthalmol. 2003;121(2):282-5.

9. Jaroudi M, Fadi M, Farah F, El Mollayess MG. Glycopyrrolatelnduced bilateral angle closure glaucoma after cervical spine surgery. Middle East Afr J Ophtalmol. 2013;20(2):182-4.

10. Tashakori A. AfshariR.Tramadol overdose as a cause of serotonin syndrome: a case series. Clin Toxicol. 2010:48:337-41.

11. Grond S, Sablotzki A. Clinical pharmacology of tramadol. ClinPharmacokinet. 2004:43:879-923.

12. Makris A, Matala ME, Tsirigotis A, Karmaniolou I. Apnea and Mydriasis after postoperativetramadol administration: an unusual complication and possible underlying mechanisms. Anaesthesia. 2012;67:73-84. 